\title{
ON THE MULTIPLE POINTS OF CERTAIN MEROMORPHIC FUNCTIONS
}

\author{
J. K. LANGLEY
}

(Communicated by Albert Baernstein II)

\begin{abstract}
We show that if $f$ is transcendental and meromorphic in the plane and $T(r, f)=o(\log r)^{2}$, then $f$ has infinitely many critical values. This is sharp. Further, we apply a result of Eremenko to show that if $f$ is meromorphic of finite lower order in the plane and $N\left(r, 1 / f f^{\prime \prime}\right)=o\left(T\left(r, f^{\prime} / f\right)\right)$, then $f(z)=\exp (a z+b)$ or $f(z)=(a z+b)^{-n}$ with $a$ and $b$ constants and $n$ a positive integer.
\end{abstract}

\section{INTRODUCTION}

If $g$ is a function transcendental and meromorphic in the plane, then the term

$$
N_{1}(r, g)=N(r, g)-\bar{N}(r, g)+N\left(r, 1 / g^{\prime}\right),
$$

in which the counting functions are defined as in [11, Chapter 2], counts the multiple points of $g$. The following has been proved by Eremenko.

Theorem A [5]. Let $g$ be transcendental and meromorphic in the plane of finite lower order such that $\delta(\infty, g)=0$ and $N_{1}(r, g)=o(T(r, g))$. Then there exist an integer $2 \rho \geq 2$ and continuous functions $L_{1}(r)$ and $L_{2}(r)$ such that $L_{1}(c t)=L_{1}(t)(1+o(1))$ and $L_{2}(c t)=L_{2}(t)+o(1)$ as $t \rightarrow+\infty$, uniformly for $1 \leq c \leq 2$, and such that

$$
-\log \left|g^{\prime}\left(r e^{i \theta}\right)\right|=\pi r^{\rho} L_{1}(\rho)\left|\cos \left(\rho\left(\theta-L_{2}(r)\right)\right)\right|+o\left(r^{\rho} L_{1}(r)\right)
$$

as $r \rightarrow+\infty$, uniformly in $\theta, 0 \leq \theta \leq 2 \pi$, provided that $r e^{i \theta}$ lies outside an exceptional set $C_{0}$ of discs $B\left(z_{k}, r_{k}\right)$ with the property that if $R$ is large, then the sum of the radii $r_{k}$ of the discs $B\left(z_{k}, r_{k}\right)$ for which $\left|z_{k}\right|<R$ is $o(R)$. Further, $\sum_{a \in \mathbb{C}} \delta(a, g)=2$ and $T(r, g)=(1+o(1)) r^{\rho} L_{1}(r)$.

It follows from Theorem A that a transcendental meromorphic function $g$ of order less than 1 cannot satisfy $N_{1}(r, g)=o(T(r, g))$ and so must have multiple points (Shea [19] had earlier proved this when $g$ has order less than $1 / 2)$. Infinitely many of these multiple points must be zeros of $g^{\prime}$, as is shown by the the following result from [6].

Received by the editors September 28, 1993.

1991 Mathematics Subject Classification. Primary 30D35. 
Theorem B. Suppose that $g$ is transcendental and meromorphic in the plane with $T(r, g)=o(r)$. Then $g^{\prime}$ has infinitely many zeros.

Further, if $g$ is transcendental meromorphic with $T(r, g)=o\left(r^{1 / 2}\right)$ or transcendental entire with $T(r, g)=o(r)$, then $g^{\prime} / g$ must have zeros. These assertions are proved in [3] and are shown there to be sharp.

This suggests the question as to whether a growth condition on a transcendental meromorphic function $f$ forces $f$ to have infinitely many critical values, that is, values take by $f$ at multiple points of $f$. If $T(r, f)=o\left(r^{1 / 2}\right)$ and $f$ is transcendental with only finitely many poles, it is easily seen from the discussion in [18, pp. 269-272] that $\infty$ must be an accumulation point of critical values of $f$, for otherwise the inverse function $f^{-1}$ would have a logarithmic singularity at $\infty$ and, if $R$ is large, there would exist a simply-connected unbounded component $U$ of the set $\{z \in \mathbb{C}:|f(z)|>R\}$, with $|f(z)|=R$ on the boundary of $U$, which contradicts the $\cos \pi \rho$ theorem [11, p. 119]. Corresponding to this remark is the obvious example $\cos (\sqrt{z})$.

The above observation and example also appear in [2], of which the author became aware after the first draft of the present paper was written. Among other results in [2] concerning asymptotic and critical values of meromorphic functions, it is shown (Corollary 3) that if the transcendental meromorphic function $f$ has finite order $\rho$ and only finitely many critical values, then the number of asymptotic values of $f$ is at most $2 \rho$.

While a transcendental entire function always has $\infty$ as an asymptotic value, by the classical theorem of Iversen [18], meromorphic functions need not have any asymptotic values at all. Bank and Kaufman [1] (see also [13, Chapter 11]) proved the existence of a function $f$ transcendental and meromorphic in the plane with $T(r, f)=O(\log r)^{2}$, satisfying the differential equation

$$
\left(z^{2}-4\right)\left(f^{\prime}(z)\right)^{2}=4\left(f(z)-e_{1}\right)\left(f(z)-e_{2}\right)\left(f(z)-e_{3}\right)
$$

in which the $e_{j}$ are distinct complex numbers, and this function $f$ clearly has just 4 critical values. This example is obtained from the Weierstrass doubly periodic function. For smaller growth, we prove the following theorem, the proof of which is based on a combination of representations for the function in annuli with the Riemann-Hurwitz formula.

Theorem 1. If $f$ is transcendental and meromorphic in the plane with $T(r, f)=$ $o(\log r)^{2}$, then $f$ has infinitely many critical values.

Our second result is a fairly straightforward application of Theorem A, coupled with a variant of a method of Mues from [17]. It was proved in [14] that if $f$ is meromorphic in the plane and $f$ and $f^{\prime \prime}$ have no zeros, then $f(z)=\exp (a z+b)$ or $f(z)=(a z+b)^{-n}$ with $a$ and $b$ constants and $n$ a positive integer. This proved a conjecture of Hayman [10,12], the case where $f$ has finite order having been settled by Mues in [17]. The same conclusion holds if $f$ is meromorphic in the plane and $N\left(r, 1 / f f^{(k)}\right)=o\left(T\left(r, f^{\prime} / f\right)\right)$ for some $k \geq 3$ [8, Theorem 2; see also 7, 9]. We prove here the following result.

Theorem 2. Suppose that $f$ is meromorphic of finite lower order in the plane and that $a_{1}$ and $a_{0}$ are rational functions such that the differential equation

$$
y^{\prime \prime}+a_{1} y^{\prime}+a_{0} y=0
$$


has linearly independent rational solutions $f_{1}$ and $f_{2}$. If $F(z)=f^{\prime \prime}(z)+$ $a_{1}(z) f^{\prime}(z)+a_{0}(z) f(z)$ and $N(r, 1 / f F)=o\left(T\left(r, f^{\prime} / f\right)\right)$, then $f^{\prime} / f$ is $r a$ tional and $f$ and $F$ have no zeros.

Corollary. If $f$ is meromorphic of finite lower order in the plane and

$$
N\left(r, 1 / f f^{\prime \prime}\right)=o\left(T\left(r, f^{\prime} / f\right)\right),
$$

then $f(z)=\exp (a z+b)$ or $f(z)=(a z+b)^{-n}$ with $a$ and $b$ constants and $n$ a positive integer.

The corollary follows at once from Theorem 2 using [11, p. 76]. Note that the assumption that (1.1) has a rational fundamental solution set implies that $a_{j}(z)=O\left(|z|^{j-2}\right)$ as $z \rightarrow \infty$ and that larger coefficients cannot be allowed in general, as the example $g(z)=\sec (\sqrt{z}), G(z)=g^{\prime \prime}(z)+(1 / 2 z) g^{\prime}(z)+$ $(1 / 4 z) g(z)=g^{3}(z) / 2 z$, shows. In [14] and [15], the author determined all functions $f$ meromorphic in the plane such that $f$ and $f^{\prime \prime}+a_{1} f^{\prime}+a_{0} f$ have only finitely many zeros, where $a_{1}$ and $a_{0}$ are rational. It seems possible that the conclusion of Theorem 2 would be true without any assumption on the growth of $f$ and with $a_{1}$ and $a_{0}$ any rational functions satisfying $a_{j}(z)=O\left(|z|^{j-2}\right.$ ) (in which case (1.1) might not have solutions meromorphic in a neighbourhood of infinity), but the present proof, which consists of applying Theorem $A$ to

$$
H(z)=\frac{f_{1}^{\prime}(z)-\left(f^{\prime}(z) / f(z)\right) f_{1}(z)}{f_{2}^{\prime}(z)-\left(f^{\prime}(z) / f(z)\right) f_{2}(z)}
$$

requires $H$ to be meromorphic in the plane of finite lower order.

\section{Preliminaries}

A key role in the proof of Theorem 1 is played by the Riemann-Hurwitz formula (see [20, Chapter 1]): Suppose that $D$ and $G$ are bounded domains of connectivity $m$ and $n$ respectively and that $f: D \rightarrow G$ is an analytic function with the property that, for any sequence $\left(z_{k}\right)$ in $D, z_{k}$ tends to the boundary $\partial D$ as $k \rightarrow \infty$ (in the sense that if $K$ is a compact subset of $D$, then $z_{k} \in D \backslash K$ for all large enough $k$ ) if and only if $f\left(z_{k}\right)$ tends to $\partial G$. Then there exists a positive integer $p$ such that all values $w$ belonging to $G$ are taken $p$ times in $D$, counting multiplicities, and such that $m-2=p(n-2)+r$, where $r$ is the number of critical points of $f$ in $D$, that is, the number of zeros of $f^{\prime}$ in $D$, counting multiplicities.

Suppose now that $f$ is a function meromorphic in the plane with only finitely many critical values. If $R$ and $S$ are large, any bounded component of the set $\{z \in \mathbb{C}: R<|f(z)|<S\}$ must be doubly-connected, while any bounded component of the set $\{z \in \mathbb{C}:|f(z)|>S\}$ contains one (possibly multiple) pole of $f$ and is simply-connected.

Lemma 1. Let $n(t)$ be nondecreasing, integer valued, and continuous from the right such that $n(1)=0$ and $n(t)=o(\log t)$ as $t \rightarrow+\infty$. Set

$$
h(r)=\int_{1}^{r} t d n(t) .
$$

If $\delta$ is a positive constant, then the set $E(\delta)=\{r \geq 1: h(r) \geq \delta r\}$ has logarithmic density 0. 
Proof. Let $\chi(t)$ be the characteristic function of $E(\delta)$, so that $\chi(t)=1$ if $t \geq 1$ and $t \in E(\delta)$ and $\chi(t)=0$ otherwise. Then

$$
\begin{aligned}
\int_{1}^{r} \chi(t) / t d t & \leq(1 / \delta) \int_{1}^{r} h(t) / t^{2} d t=(1 / \delta) \int_{1}^{r} 1 / t d h(t)-h(r) / \delta r \\
& \leq(1 / \delta) \int_{1}^{r} d n(t)=o(\log r),
\end{aligned}
$$

which is precisely what is asserted in the lemma.

The next lemma is part of a special case of the lemma from [16].

Lemma A. Let $m(t)$ be nondecreasing, integer-valued and continuous from the right, with $m(1)=0$ and $m(t)=O(t)$ as $t \rightarrow+\infty$. Let $M>3$ be a constant. Then there exists a set $E_{M}$ of lower logarithmic density at least $1-3 / M$; that is,

$$
\int_{1}^{r} \chi(t) / t d t>(1-3 / M-o(1)) \log r \text { as } r \rightarrow+\infty,
$$

with $\chi(t)$ the characteristic function of $E_{M}$, such that, for $r \in E_{M}$ and $t \geq r$, we have $m(t) / m(r) \leq(t / r)^{4 M}$.

Lemma 2. Let $f$ be transcendental and meromorphic in the plane with $T(r, f)$ $=o(\log r)^{2}$. Then there exist sequences $R_{\nu}$ and $S_{\nu}$ tending to $+\infty$, nonzero constants $C_{\nu}$ and $D_{\nu}$, and integers $m_{\nu}$ and $n_{\nu}$ such that for

$$
R_{\nu} S_{\nu}^{-1} \leq|z| \leq R_{\nu} S_{\nu}
$$

we have

$$
f(z)=C_{\nu} z^{m_{\nu}}(1+o(1))
$$

and

$$
f^{\prime}(z)=D_{\nu} z^{n_{\nu}}(1+o(1)) .
$$

Proof. We write $f(z)=U(z) F(z)$ and $f^{\prime}(z)=V(z) G(z)$ where $U$ and $V$ are rational functions and $F$ and $G$ satisfy $F(0)=G(0)=1$ and have no zeros or poles in $|z| \leq 1$. We choose a small positive $\delta$ and apply Lemma 1 with $n(t)=n(t, 1 / F)+n(t, F)+n(t, 1 / G)+n(t, G)=O\left(T\left(t^{2}, f\right) / \log t\right)=o(\log t)$. Further, we apply Lemma 2 with $M=100$ and $m(t)=2^{n(t)}$. This gives arbitrarily large $r$ such that

$$
h(r)=\int_{1}^{r} t d n(t)<\delta r
$$

and, for $t \geq r$,

$$
n(t)-n(r) \leq M_{1} \log (t / r),
$$

where $M_{1}=400 / \log 2$. Since $n(t)$ is integer-valued, (2.4) implies that $f$ and $f^{\prime}$ have no zeros or poles in $\delta r \leq|z| \leq r$. Suppose that

$$
\delta^{3 / 4} r \leq|z| \leq \delta^{1 / 4} r .
$$

We write $F(z)=F_{1}(z) / f_{2}(z)$ with the $f_{j}$ entire and $f_{1}(z)=\prod_{j=1}^{\infty}\left(1-z / a_{j}\right)$, where the $a_{j}$ are the zeros of $f$ in $1<|z|<\infty$, repeated according to multiplicity. For $z$ as in (2.6) we have

$$
f_{1}(z)=z^{n(r, 1 / F)} \prod_{1}\left(-1 / a_{j}\right) \prod_{1}\left(1-a_{j} / z\right) \prod_{2}\left(1-z / a_{j}\right),
$$


in which $\prod_{1}$ denotes the product over all $a_{j}$ with $\left|a_{j}\right|<r$ and $\prod_{2}$ denotes the product over the remaining $a_{j}$. With $\sum_{1}$ defined analogously to $\prod_{1}$, we have, using (2.4),

$$
\begin{aligned}
\left|\prod_{1}\left(1-a_{j} / z\right)-1\right| & \leq \exp \left(\sum_{1}\left|a_{j} / z\right|\right)-1 \\
& \leq \exp (h(r) /|z|)-1 \leq \exp (\delta r /|z|)-1 \\
& \leq \exp \left(\delta^{1 / 4}\right)-1 .
\end{aligned}
$$

Further, (2.5) gives $n(t, 1 / f)-n(r, 1 / f) \leq M_{1}(\log (t / r))$ for $t \geq r$, and we have

$$
\begin{aligned}
\left|\prod_{2}\left(1-z / a_{j}\right)-1\right| & \leq \exp \left(|z| \int_{r}^{\infty} \frac{1}{t} d n(t, 1 / f)\right)-1 \\
& =\exp \left(|z| \int_{r}^{\infty}[n(t, 1 / f)-n(r, 1 / f)] d t / t^{2}\right)-1 \\
& \leq \exp \left(|z| M_{1} \int_{r}^{\infty} \log (t / r) d t / t^{2}\right)-1 \\
& =\exp \left(|z| M_{1} / r\right)-1 \leq \exp \left(M_{1} \delta^{1 / 4}\right)-1 .
\end{aligned}
$$

Now if $\varepsilon>0$ is given, we need only choose $\delta$ small enough, and (2.7), (2.8), and (2.9) then give $f_{1}(z)=\prod_{1}\left(-1 / a_{j}\right) z^{n(r, 1 / F)}(1+\rho(z))$, where $|\rho(z)|<\varepsilon$ for $z$ satisfying (2.6). Estimating $f_{2}$ in the same way gives (2.2) and the proof of (2.3) is identical.

The following is Lemma III of [4].

Lemma B. Suppose that $g$ is meromorphic in $|z| \leq R, 1<r<R$, and that $I(r)$ is a measurable subset of $[0,2 \pi]$ of measure $\mu(r)$. Then

$$
\frac{1}{2 \pi} \int_{I(r)} \log ^{+}\left|g\left(r e^{i \theta}\right)\right| d \theta \leq 11 R(R-r)^{-1} \mu(r)\left(1+\log +\frac{1}{\mu(r)}\right) T(R, g) .
$$

\section{Proof of Theorem 1}

Suppose that $f$ is transcendental and meromorphic in the plane such that $T(r, f)=o(\log r)^{2}$ and $f$ has only finitely many critical values. By the remark in the introduction we can assume that $f$ has no Picard value. Let $R_{\nu}, S_{\nu}$, $C_{\nu}, D_{\nu}, m_{\nu}, n_{\nu}$ be as in Lemma 2. We can assume that, as $\nu \rightarrow \infty$,

$$
\left|C_{\nu}\right| R_{\nu}^{m_{\nu}} \rightarrow \alpha, \quad 1 \leq \alpha \leq+\infty,
$$

by taking a subsequence and replacing $f$ by $1 / f$, if necessary. We consider a number of cases.

Case 1. Suppose that $\alpha=+\infty$ and $m_{\nu}=0$ for infinitely many $\nu$.

Taking a further subsequence if necessary we can assume that

$$
100\left|C_{\nu-1}\right| R_{\nu-1}^{m_{\nu-1}}<\left|C_{\nu}\right|<100^{-1}\left|C_{\nu+1}\right| R_{\nu+1}^{m_{\nu+1}} .
$$

Take a small positive $\varepsilon$. Now (3.2) implies that if $\nu$ is large enough, the circle $|z|=R_{\nu}$ lies inside a bounded component of the set $\left\{z:\left|f(z)-C_{\nu}\right|<\varepsilon\left|C_{\nu}\right|\right\}$. This component contains no multiple point of $f$ and is multiply connected, by (3.2), which contradicts the Riemann-Hurwitz formula. 
Case 2. Suppose that $\alpha=+\infty$ and that $m_{\nu} \neq 0$ for all large $\nu$.

Then (2.2) implies that the annulus $(1 / 4) R_{\nu}<|z|<4 R_{\nu}$ contains a closed level curve $\Gamma_{\nu}$ on which $|F(z)|=k_{\nu}=\left|C_{\nu}\right| R_{\nu}^{m_{\nu}}$, and this level curve $\Gamma_{\nu}$ must be a simple closed curve winding once around the origin. We take $\mu<\nu$ such that $100 k_{\mu}<k_{\nu}$ and such that the region $U$ lying between $\Gamma_{\mu}$ and $\Gamma_{\nu}$ contains at least one zero of $f$.

Let $V_{1}$ be a component of the set $\left\{z \in U:|f(z)|<k_{\mu}\right\}$. Since $|f(z)| \geq k_{\mu}$ on $\Gamma_{\mu}$ and $\Gamma_{\nu}$, we have $|f(z)|=k_{\mu}$ on the boundary $\partial V_{1}$, which is contained in the closure $\bar{U}$ of $U$ and consists of disjoint smooth simple closed curves. Let $\gamma_{1}$ be the unique component of $\partial V_{1}$ which forms the boundary of an unbounded component of $\mathbb{C} \backslash V_{1}$, and suppose first that the winding number $n\left(\gamma_{1}, 0\right)=0$.

Now $\gamma_{1}$ cannot coincide with $\Gamma_{\mu}$, since the interior of $\Gamma_{\mu}$ is bounded, and so $\gamma_{1}$ does not meet $\Gamma_{\mu}$, using the fact that $f$ has no critical values on $|w|=k_{\mu}$. Thus $\gamma_{1}$ forms part of the boundary of a component $V^{*}$ of the set $\left\{z \in U: K_{\mu}<|f(z)|<k_{\nu}\right\}$. On $\partial V^{*}$ we have $|f(z)|=k_{\mu}$ or $|f(z)|=k_{\nu}$, and $V^{*}$ must be doubly-connected, by the Riemann-Hurwitz formula. The other component $\gamma_{2}$ of $\partial V^{*}$ must close in $\bar{U}$ and cannot coincide with $\Gamma_{\nu}$, since $V^{*}$ is doubly-connected and since there exist points arbitrarily close to $\Gamma_{\mu}$ at which $|f(z)|<k_{\mu}$. Thus $\gamma_{2}$ cannot meet $\Gamma_{\nu}$. Further, on $\gamma_{2}$ we have $|f(z)|=k_{\nu}$, and $\gamma_{2}$ forms part of the boundary of a component $V^{* *}$ of the set $\left\{z \in U:|f(z)|>k_{\nu}\right\}$, on the boundary of which $|f(z)|=k_{\nu}$. The Riemann-Hurwitz formula now implies that $V^{* *}$ is simply-connected, which is a contradiction, since $V^{*}$ lies in a bounded component of $\mathbb{C} \backslash V^{* *}$.

This contradiction proves that $n\left(\gamma_{1}, 0\right) \neq 0$. Thus $\Gamma_{\mu}$ lies in a bounded component of $\mathbb{C} \backslash V_{1}$, and $\partial V_{1}$ must have precisely two components $\omega_{1}$ and $\omega_{2}$ such that $n\left(\omega_{j}, 0\right) \neq 0$, and this is true for every component of the set $\{z \in$ $\left.U:|f(z)|<k_{\mu}\right\}$. If these components are $V_{1}, \ldots, V_{p}$ and if $p>1$, we can assume that, for each $j, V_{j}$ lies in the same component of $\mathbb{C} \backslash V_{j+1}$ as $\Gamma_{\mu}$. But then components of $\partial V_{1}$ and $\partial V_{2}$ together bound a doubly-connected region on which $|f(z)| \geq k_{\mu}$. This region must contain a pole of $f$ by the maximum principle, and the fact that it is not simply-connected contradicts the RiemannHurwitz formula. Therefore $p=1$, which is a contradiction since we can choose $\nu$ arbitrarily large.

Case 3. Suppose that $\alpha$ is finite in (3.1).

If $m_{\nu} \neq 0$ for infinitely many $\nu$, then since $S_{\nu} \rightarrow \infty$ we can take a subsequence and obtain level curves $\Gamma_{\nu}$ on which $|f(z)|=k_{\nu} \rightarrow \infty$, by considering $f(z)$ on $|z|=R_{\nu} S_{\nu}^{ \pm 1 / 4}$, and then argue as in Case 2. We assume henceforth that $m_{\nu}=0$ for all large $\nu$, so that without loss of generality

$$
f(z)=1+o(1), \quad R_{\nu} S_{\nu}^{-1} \leq|z| \leq R_{\nu} S_{\nu} .
$$

We also have (2.3), which we write in the form

$$
f^{\prime}(z)=D_{\nu} z^{n_{\nu}}(1+\delta(z)), \quad \delta(z)=o(1), \quad R_{\nu} S_{\nu}^{-1} \leq|z| \leq R_{\nu} S_{\nu},
$$

and we can assume that $\delta^{\prime}(z)=o(1 /|z|)$ for the same range of values of $z$, because otherwise we can replace $S_{\nu}$ by $S_{\nu}^{1 / 2}$ and apply Cauchy's estimate for derivatives. 
Now if $n_{\nu} \leq-2$ in (3.4), then integration by parts gives, with $q_{\nu}=n_{\nu}+1$, $E_{\nu}=D_{\nu} q_{\nu}^{-1}, z_{0}=R_{\nu} S_{\nu}$, and $L_{\nu}$ a constant, the estimates

$$
f(z)=L_{\nu}+E_{\nu} z^{q_{\nu}}(1+\delta(z))-\int_{z_{0}}^{z} E_{\nu} t^{q_{\nu}} \delta^{\prime}(t) d t=L_{\nu}+E_{\nu} z^{q_{\nu}}(1+o(1)) .
$$

In obtaining the last estimate of (3.5) we have taken the path of integration to be the straight line segment from $z_{0}$ to $|z|$, followed by part of the circle $|t|=|z|$.

If $n_{\nu}=-1$ in (3.4), then the integral of $f^{\prime}(z)$ around the circle $|z|=R_{\nu}$ will be $D_{\nu}(2 \pi i+o(1))$, which is clearly impossible. Finally if $n_{\nu} \geq 0$ in (3.4), we take $z_{0}=R_{\nu} S_{\nu}^{-1}$ and obtain (3.5) again.

Again we consider cases.

Case A. Suppose that $\left|1-L_{\nu}\right| \geq(1 / 4)\left|E_{\nu}\right| R_{\nu}^{q_{\nu}}$ for infinitely many $\nu$.

In this case, since $q_{\nu} \neq 0$ we find, using (3.3), that

$$
f(z)-1=\left(L_{\nu}-1\right)(1+o(1))=o(1)
$$

either on $R_{\nu} S_{\nu}^{-1} \leq|z| \leq R_{\nu} S_{\nu}^{-1 / 2}$ or on $R_{\nu} S_{\nu}^{1 / 2} \leq|z| \leq R_{\nu} S_{\nu}$, and we can apply the reasoning of Case 1 to $g(z)=1 /(f(z)-1)$.

Case B. Suppose that $\left|1-L_{\nu}\right|<(1 / 4)\left|E_{\nu}\right| R_{\nu}^{q_{\nu}}$ for all large $\nu$.

Again, since $q_{\nu} \neq 0$, we can obtain, on a smaller annulus formed as in Case A, the estimate $f(z)-1=E_{\nu} z^{q_{\nu}}(1+o(1))$, and on these annuli $E_{\nu} z^{q_{\nu}} \rightarrow 0$ uniformly, by (3.3). Thus we may apply the reasoning of Case 2 to $g(z)=$ $1 /(f(z)-1)$.

\section{Proof of Theorem 2}

Let $f_{1}$ and $f_{2}$ be linearly independent rational solutions of (1.1), so that the Wronskian $W\left(f_{1}, f_{2}\right)=W$ is also rational. Now $\left(f_{2} / f_{1}\right)^{\prime}=W f_{1}^{-2}=$ $d z^{q-1}(1+o(1))$ as $z \rightarrow \infty$, for some nonzero constant $d$ and integer $q$, and $q$ cannot be zero, since $f_{2} / f_{1}$ is by assumption rational. Therefore we may assume that $f_{2}(z) / f_{1}(z)=z^{q}(1+o(1))$ as $z \rightarrow \infty$ and that $q$ is positive.

Assuming that $f$ and $F$ are as in the statement of Theorem 2, and that $N(r, 1 / f F)=o\left(T\left(r, f^{\prime} / f\right)\right)$ and $f^{\prime} / f$ is transcendental, we set

$$
H(z)=K_{1}(z) / K_{2}(z), \quad K_{j}(z)=f_{j}^{\prime}(z)-f_{j}(z) f^{\prime}(z) / f(z),
$$

so that $H$ is transcendental of finite lower order.

Now all but finitely many poles of $H$ are zeros of $K_{2}$ which are not zeros or poles of $f$. Further, $K_{j}^{\prime}(z)=-f_{j}(z) F(z) / f(z)-K_{j}(z)\left(a_{1}(z)+f^{\prime}(z) / f(z)\right)$, so that at a zero $z$ of $K_{2}$ with $z$ large and with multiplicity $m \geq 2, F(z)$ must have a zero of multiplicity $m-1$. Thus $N(r, H)-\bar{N}(r, H) \leq N(r, 1 / F)+$ $O(\log r)=o(T(r, H))$, using (4.1). Moreover,

$$
H^{\prime}(z)=-W(z) F(z) / f(z) K_{2}(z)^{2},
$$

so that zeros $z$ of $H^{\prime}$ with $z$ large can only occur at zeros of $F$ or at simple zeros of $f$, which implies that $N\left(r, 1 / H^{\prime}\right)=o(T(r, H))$. We may therefore apply Theorem A to $g(z)$, where $g(z)$ is either $H(z)$ or $1 /(b-H(z))$, for some constant $b, g$ being normalized so that $\delta(\infty, g)=0$.

We take a small positive constant $\varepsilon$ and a sequence $\left(r_{k}\right)$ such that $r_{0}$ is large and $2 r_{k} \leq r_{k+1} \leq 4 r_{k}$ for each $k \geq 0$ and such that the circles $|z|=r_{k}$ do not 
meet the exceptional set $C_{0}$ of Theorem $A$ and further such that $T\left(r_{k}, f^{\prime} / f\right) \leq$ $O\left(T\left(r_{k}, f\right)\right)$ for each $k$. Now $L_{2}(r)=L_{2}\left(r_{k}\right)+o(1)$, uniformly for $r_{k} \leq r \leq$ $r_{k+1}$. For each integer $k \geq 0$ we choose $\theta_{k}^{*}$ in $\left[L_{2}\left(r_{k}\right)-\pi / 16 \rho, L_{2}\left(r_{k}\right)+\pi / 16 \rho\right]$ such that the straight line segments $z=r \exp \left(i\left(\theta_{k}^{*}+j \pi / \rho\right)\right), r_{k} \leq r \leq r_{k+1}$, $0 \leq j \leq 2 \rho-1$, do not meet $C_{0}$. Obviously, $\left|\cos \left(\rho\left(\theta_{k}^{*}-L_{2}\left(r_{k}\right)\right)\right)\right| \geq 3 / 4$. For each integer $j$ with $0 \leq j \leq 2 \rho-1$ we then choose $\Gamma_{j}$ to be the union of the straight line segments $z=r \exp \left(i\left(\theta_{k}^{*}+j \pi / \rho\right)\right), r_{k} \leq r \leq r_{k+1}, k \geq 0$, and the $\operatorname{arcs} z=r_{k} \exp (i \theta),\left|\theta-L_{2}\left(r_{k}\right)-j \pi / \rho\right| \leq \pi / 2 \rho-\varepsilon$. On $\Gamma_{j}$, Theorem A gives $\left|g^{\prime}(z)\right| \leq|z|^{-3 N}$, where $N$ is a large positive integer, so $\left|g(z)-A_{j}\right|=O\left(|z|^{-2 N}\right)$, for some constant $A_{j}$. In fact a much stronger estimate is proved in [5], but this suffices for our purposes here and gives either $\left|H(z)-B_{j}\right|=O\left(|z|^{-2 N}\right)$, for some constant $B_{j}$, or $1 / H(z)=O\left(|z|^{-2 N}\right)$. Thus either $G(z)=H(z) f_{2}(z) / f_{1}(z)$ or $G(z)^{-1}$ is $O(1 /|z|)$ on $\Gamma_{j}$, and in either case we obtain there $f^{\prime}(z) / f(z)=$ $O(1 /|z|)$, so $\log ^{+}|1 / f(z)|=O(\log |z|)$. Now Lemma B implies that for some small constant $\delta$, which satisfies $\delta=O(\varepsilon \log (1 / \varepsilon))$, we have, for each $k \geq 0$,

$$
\begin{aligned}
T\left(r_{k}, f\right) & \leq(1+o(1)) m\left(r_{k}, 1 / f\right) \\
& \leq(\delta / 2) T\left(2 r_{k}, 1 / f\right)+O\left(\log r_{k}\right) \leq \delta T\left(r_{k+1}, f\right),
\end{aligned}
$$

so, for some positive constant $c$, independent of $\delta$, and for $r_{k} \leq r \leq r_{k+1}$,

$$
T(r, f) \geq T\left(r_{k}, f\right) \geq \delta^{-k} T\left(r_{0}, f\right) \geq \delta^{-2 c \log r_{k}} T\left(r_{0}, f\right) \geq \delta^{-c \log r} T\left(r_{0}, f\right),
$$

which contradicts the assumption that $f$ has finite lower order and proves Theorem 2.

\section{REFERENCES}

1. S. Bank and R. Kaufman, On meromorphic solutions of first-order differential equations, Comment. Math. Helv. 51 (1976), 289-299.

2. W. Bergweiler and A. Eremenko, On the singularities of the inverse to a meromorphic function of finite order, preprint.

3. J. Clunie, A. Eremenko, and J. Rossi, On equilibrium points of logarithmic and Newtonian potentials, J. London Math. Soc. (2) 47 (1993), 309-320.

4. A. Edrei and W. H. J. Fuchs, Bounds for the number of deficient values of certain classes of meromorphic functions, Proc. London Math. Soc. (3) 12 (1962), 315-344.

5. A. Eremenko, Meromorphic functions with small ramification, Indiana Univ. Math. J. 42 (1994), 1193-1218.

6. A. Eremenko, J. K. Langley, and J. Rossi, On the zeros of meromorphic functions of the form $\sum_{k=1}^{\infty} a_{k} /\left(z-z_{k}\right)$, J. Analyse Math. 62 (1994), 271-286.

7. G. Frank, Eine Vermutung von Hayman über Nullstellen meromorpher Funktionen, Math. Z. 149 (1976), 29-36.

8. G. Frank and S. Hellerstein, On the meromorphic solutions of nonhomogeneous linear differential equations with polynomial coefficients, Proc. London Math. Soc. (3) 53 (1986), 407-428.

9. G. Frank, W. Hennekemper, and G. Polloczek, Über die Nullstellen meromorpher Funktionen and ihrer Ableitungen, Math. Ann. 225 (1977), 145-154.

10. W. K. Hayman, Picard values of meromorphic functions and their derivatives, Ann. of Math. (2) 70 (1959), 9-42.

11. _ Meromorphic functions, Clarendon Press, Oxford, 1964.

12. - Research problems in function theory, Athlone Press, London, 1967.

13. I. Laine, Nevanlinna theory and complex differential equations, de Gruyter Stud. Math., vol. 15, de Gruyter Studies in Mathematics, de Gruyter, Berlin, 1993. 
14. J. K. Langley, Proof of a conjecture of Hayman concerning $f$ and $f^{\prime \prime}$, J. London Math. Soc. (2) 48 (1993), 500-514.

15. __ On second order linear differential polynomials, Resultate Math. 26 (1994), 51-514.

16. J. Miles and J. Rossi, Linear combinations of logarithmic derivatives of entire functions with applications to differential equations, preprint.

17. E. Mues, Über eine Vermutung von Hayman, Math. Z. 119 (1972), 11-20.

18. R. Nevanlinna, Eindeutige analytische Funktionen, Springer, Berlin, 1936.

19. D. Shea, On the frequency of multiple values of a meromorphic function of small order, Michigan Math. J. 32 (1985), 109-116.

20. N. Steinmetz, Rational iteration, de Gruyter Stud. Math., vol. 16, de Gruyter Studies in Mathematics, de Gruyter, Berlin and New York, 1993.

Department of Mathematics, University of Nottingham, Nottingham NG7 2RD, ENGLAND

E-mail address: jkl@maths.nott.ac.uk 